\title{
Rolling and Lifting Probabilities for Sediment Entrainment
}

\author{
Fu-Chun $\mathrm{Wu}^{1}$ and Yi-Ju Chou ${ }^{2}$
}

\begin{abstract}
This study addresses the rolling and lifting probabilities for sediment entrainment by incorporating the probabilistic features of the turbulent fluctuation and bed grain geometry. The lognormally distributed instantaneous velocity and uniformly distributed initial grain position, along with a relation between lift coefficient and particle Reynolds number, are used to extend the theoretical formulation of the entrainment probabilities in smooth-bed flows. The two threshold conditions identified herein enable us to precisely define the probabilities of entrainment in the rolling and lifting modes. The results obtained in this study coincide well with the published data. The lifting probability increases monotonously with the dimensionless shear stress $\theta$, which is consistent with the earlier results yet displays improved agreement with the experimental data. The maximum value of rolling probability, with a magnitude of 0.25 , occurs at $\theta$ $\approx 0.15$. For $\theta<0.05$ (or $\theta>0.6$ ), the rolling (or lifting) probability makes up more than $90 \%$ of the total entrainment probability and thus can be used as an approximation to the total probability of entrainment. The proposed rolling and lifting probabilities are further linked to the two separate criteria for incipient motion to explore the critical entrainment probabilities. The results reveal that a consistent probability corresponding to the critical state of sediment entrainment cannot be found.
\end{abstract}

DOI: 10.1061/(ASCE)0733-9429(2003)129:2(110)

CE Database keywords: Entrainment; Sediment; Probability distribution; Drag coefficient; Lift coefficients.

\section{Introduction}

Sediment entrainment is defined as the transition from repose to displacement. Previous studies (e.g., Halow 1973; Drake et al. 1988) indicated that the entrainment of sediment particles occurs in four different ways, namely, rolling, sliding, lifting, and bouncing (or impact ejection). The initiation of particle motion by sliding or bouncing occurs only rarely and is much less important than the other two modes, thus often neglected in the analysis (details see Halow 1973; Drake et al. 1988; Ling 1995). The entrainment of sediment has been investigated for over eight decades from various viewpoints (reviewed by Buffington and Montgomery 1997). One approach is to determine the critical shear stress for incipient motion of sediment. The work of Shields (1936) is probably the most well-known entrainment criterion that falls into this category. Quantification of the threshold shear stress is the basis for prediction of transport rate in many bed load equations (e.g., Meyer-Peter and Müller 1948; Parker 1979; among others). Lately, Ling (1995) further derived the rolling and lifting criteria for incipient motion of spherical sediment particles. His results reveal that the lifting threshold is consistently higher than the rolling threshold in the hydraulically smooth and transitional flow regimes. On the other hand, some researchers believed the existence of a range of threshold values for initial movement

\footnotetext{
${ }^{1}$ Associate Professor, Dept. of Bioenvironmental Systems Engineering and Hydrotech Research Institute, National Taiwan Univ., Taipei 106, Taiwan, Republic of China. E-mail: fcwu@hy.ntu.edu.tw

${ }^{2}$ Research Assistant, Dept. of Bioenvironmental Systems Engineering, National Taiwan University, Taipei 106, Taiwan, Republic of China.

Note. Discussion open until July 1, 2003. Separate discussions must be submitted for individual papers. To extend the closing date by one month, a written request must be filed with the ASCE Managing Editor. The manuscript for this paper was submitted for review and possible publication on September 13, 2001; approved on May 30, 2002. This paper is part of the Journal of Hydraulic Engineering, Vol. 129, No. 2, February 1, 2003. @ASCE, ISSN 0733-9429/2003/2-110-119/\$18.00.
}

and thus employed the probabilistic model as an alternative approach to the sediment entrainment problem (e.g., Einstein 1942; Grass 1970; Gessler 1971) and the prediction of bed load transport (e.g., Einstein 1950; Paintal 1971; Sun and Donahue 2000). The field and laboratory observations reported in several recent works (e.g., Lavelle and Mofjeld 1987; Kirchner et al. 1990; Buffington et al. 1992; Wilcock et al. 1996) also confirmed the variability of critical shear stress that could be attributed to a number of random factors. The contributors to the stochastic nature of sediment entrainment include the temporal fluctuations of turbulent flow (Kalinske 1947), heterogeneities in grain size, shape, and density (Bridge and Bennett 1992), bed grain geometry (Naden 1987), availability of sediment (Church 1978), exposure and sheltering effect (McEwan and Heald 2001), and bed roughness (Papanicolaou et al. 2001), just to mention a few among many others.

The probabilistic approach to modeling sediment transport has been showing promise, however, a variety of entrainment probabilities was used for developing bed load formulas. For example, Einstein (1950) used the lifting (pickup) probability to derive the bed load function; Sun and Donahue (2000) employed the rolling probability in their fractional bed load equation, whereas Paintal (1971) used the sliding probability in his bed load model. Cheng and Chiew (1998) presented a theoretical formulation of the lifting probability for sediment entrainment, which was later modified by Wu and Lin (2002). Both of their works incorporated the probability distribution of instantaneous velocity to explore the relationship between lifting probability and flow condition. The Gaussian and log-normal distributions of instantaneous velocity were adopted in their analyses, respectively. Their optimal choices of a constant lift coefficient were based on the best fitting to the experimental data but not to vary as a function of the flow condition. Moreover, the sediment particle was assumed to lie on a bed of closely packed particles yet such a configuration represented only one of the many possible situations. Papanicolaou et al. (2002) provided a quantitative model for predicting sedi- 


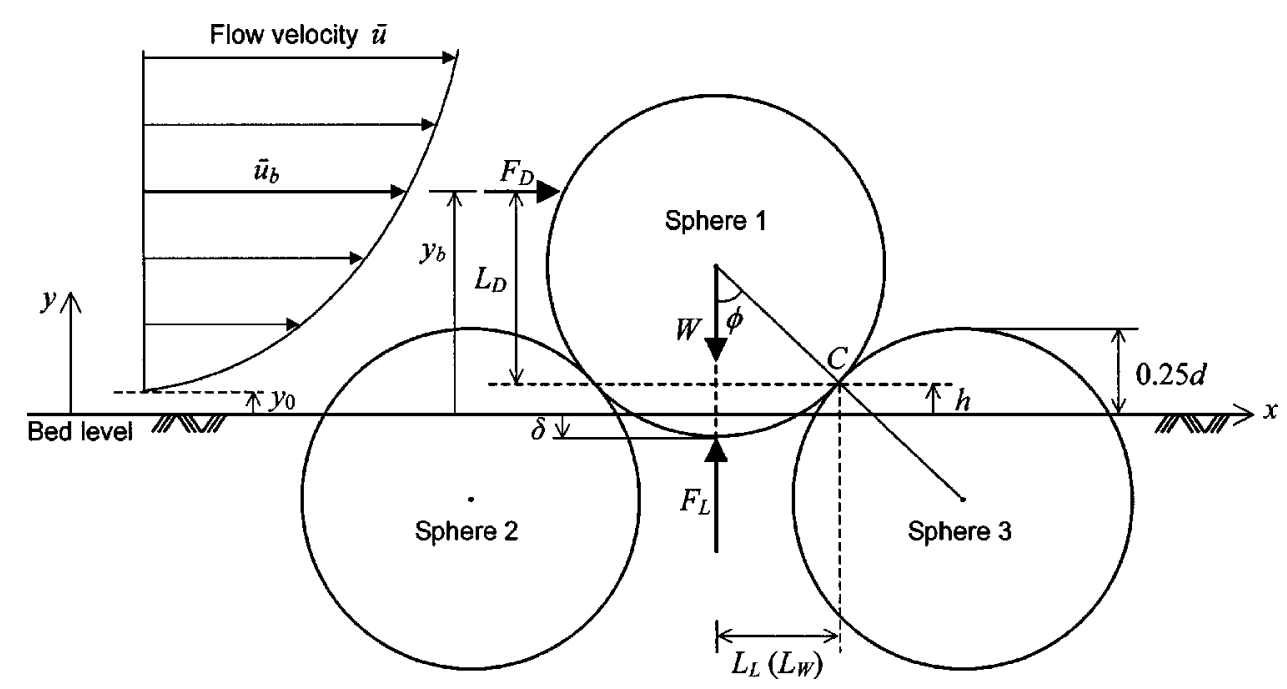

Fig. 1. Definition sketch showing longitudinal section ( $x-y$ plane) of bed grain geometry, flow velocity, and forces acting on Sphere 1

ment entrainment under three representative bed packing densities corresponding to the isolated, wake interference, and skimming flow regimes. However, further studies incorporating more general considerations can be conducted to modify the formulation of entrainment probability. The purpose of this study is to develop theoretical components for evaluating two types of entrainment probability, i.e., the rolling and lifting probabilities, in hydraulically smooth-bed and transitional open-channel flows. The thresholds for two different entrainment modes are identified, which lead to a more precise definition of the rolling and lifting probabilities. The present study extends the previous work of Wu and Lin (2002) in the sense that both the fluctuation of turbulent flow and the randomness of bed grain geometry are considered in the derivation of rolling and lifting probabilities. Also taken into consideration is the dependence of the lift coefficient on particle Reynolds number. The proposed lifting and rolling probabilities are then verified with the published data. These two probabilities are further incorporated with the rolling and lifting thresholds developed by Ling (1995) to demonstrate the inconsistency involved in the calculation of critical shear stress.

\section{Theoretical Components}

\section{Bed Grain Geometry}

Consider a spherical particle of size $d$ resting on the bed consisting of identical spheres (longitudinal section shown in Fig. 1).
The schematization is three dimensional, i.e., $x, y$, and $z$ axes represent the longitudinal, vertical, and transverse directions, respectively. The theoretical bed level $(y=0)$, where flow velocity is zero, is set to be located at a distance below the top of the bed grains. A range of values have been used in the literature indicating that the bed level is commonly taken as $0.15 d-0.3 d$ below the top of the sand-grain roughness (Bridge and Bennett 1992; Nezu and Nakagawa 1993). To be consistent with the preceding work of this paper (van Rijn 1984; Cheng and Chiew 1998; Wu and Lin 2002), a distance of $0.25 d$ is adopted in the present study. As illustrated in Fig. 1, Sphere 1 is in contact with an upstream and a downstream bed particle (Spheres 2 and 3, respectively). The point of contact between Spheres 1 and 3 (labeled as $C$ ) is located at a distance of $h$ from the bed level, while the bottom of Sphere 1 is at the position with a distance of $\delta$ from the bed level (note that both $h$ and $\delta$ are positive upwards and negative downwards). The lower and upper limits of $\delta$ are shown in Figs. 2(a and b), respectively. When $\delta=-0.75 d$, Sphere 1 is at the lowest-possible position to protrude into the flow; when $\delta$ $=0.116 \mathrm{~d}$, Sphere 1 is resting at the highest-possible position to remain stable. The initial position of Sphere 1 is supposed to be randomly oriented relative to the bed level, thus $\delta$ is treated as a random variable. Following the encouraging results of Paintal (1971) and $\mathrm{Wu}$ et al. (2000), we accept that $\delta$ is uniformly distributed. Although a near-normal distribution with positive skewness has been suggested as a first approximation to the gravel-bed topography (Nikora et al. 1998), more research is still needed to (a)

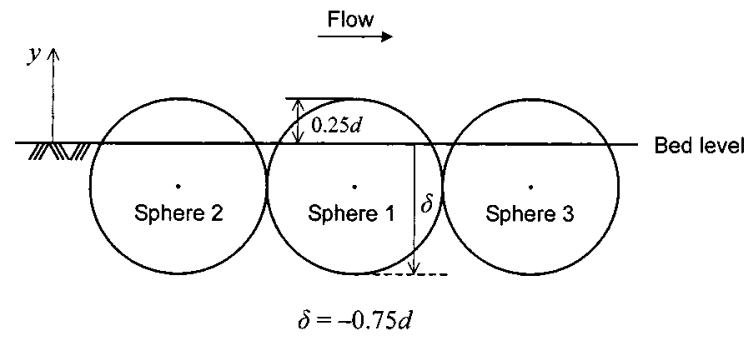

(b)

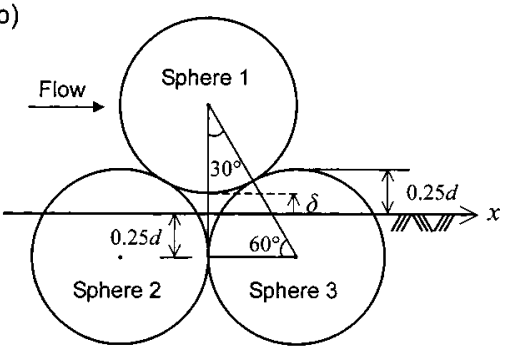

$\delta=0.116 d$

Fig. 2. Longitudinal diagram ( $x-y$ plane) demonstrating (a) lower limit and (b) upper limit of $\delta$ 


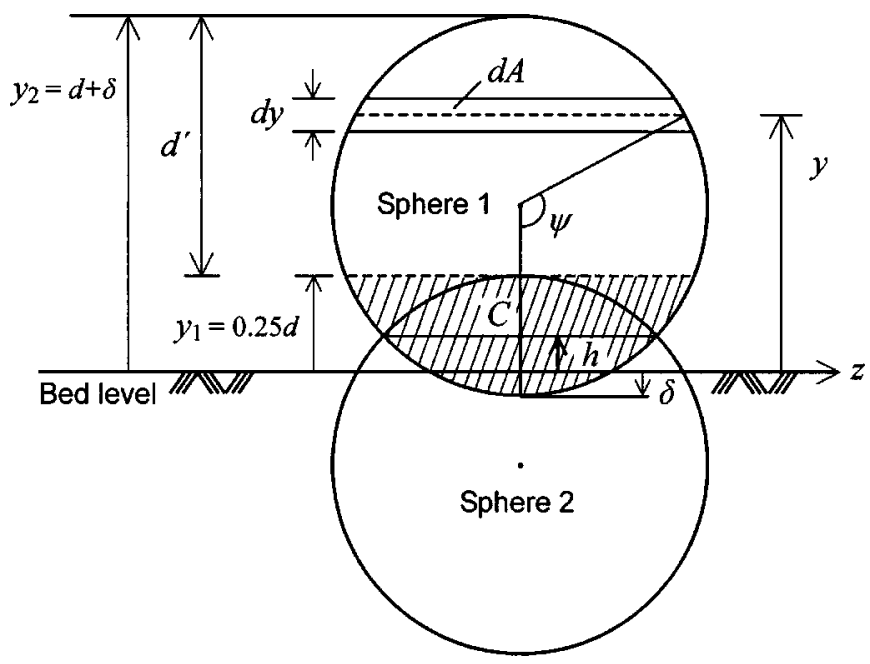

Fig. 3. Definition sketch showing transverse section ( $y-z$ plane) of exposed bed grain (view into flow)

test its validity on the smooth and transitional beds. The probability density function (pdf) of $\delta$ is thus expressed as

$$
\begin{aligned}
& f_{\Delta}(\delta)=\frac{1}{0.116 d-(-0.75 d)}=\frac{1}{0.866 d}, \\
& \text { for }-0.75 d \leqslant \delta \leqslant 0.116 d
\end{aligned}
$$

\section{Thresholds for Entrainment}

The incipient motion of sediment occurs when the stability of a particle is disturbed. Such instability can be attributed to the imbalance of forces or force moments caused by the forces exerted on the particle in the flow (see Ling 1995). Fig. 1 shows that the external forces acting on Sphere 1 include the submerged weight $(W)$ and the instantaneous hydrodynamic forces, which can be resolved into a drag force $\left(F_{D}\right)$ and a lift force $\left(F_{L}\right)$. Various forms of $F_{L}$ can be found in the literature (e.g., Naden 1987; Ling 1995); here we follow Cheng and Chiew $(1998,1999)$ to use the conventional expressions for these forces, i.e.,

$$
\begin{gathered}
W=\left(\rho_{s}-\rho\right) g \frac{\pi d^{3}}{6} \\
F_{D}=C_{D} \frac{\rho A u_{b}^{2}}{2} \\
F_{L}=C_{L} \frac{\rho A u_{b}^{2}}{2}
\end{gathered}
$$

where $\rho$ and $\rho_{s}=$ densities of fluid and sediment particle, respectively; $g=$ gravitational acceleration; $A=$ frontal area exposed to the flow, shown in Fig. $3(y-z$ plane across the flow) as the noncrosshatched frontal area of Sphere $1 ; u_{b}=$ area-averaged instantaneous velocity, defined as the averaging of streamwise velocity over the exposed frontal area $A$ (which will be specified later in this section); $C_{D}$ and $C_{L}=$ drag and lift coefficients, respectively. The instantaneous velocity $u_{b}$ is used in Eq. (2) to account for the effect of fluctuating fluid forces. The drag coefficient of a sphere is known to depend upon flow conditions and can be obtained by the following formula (Schiller and Naumann 1933):

$$
C_{D}=\left(24 / \mathrm{R}_{p}\right)\left(1+0.15 \mathrm{R}_{p}^{0.687}\right)
$$

where $\mathrm{R}_{p}=$ particle Reynolds number defined as $\bar{u}_{b} d^{\prime} / \nu$, in which $\bar{u}_{b}=$ area-averaged temporal mean velocity, $d^{\prime}=$ exposed diameter of a particle (shown in Fig. 3 ) $=0.75 d+\delta$, $v=$ kinematic viscosity of fluid. Eq. (3) is valid for $\mathrm{R}_{p} \leqslant 1,754$, while $C_{D}=0.36$ for $\mathrm{R}_{p}>1,754$ in smooth-bed flows (Graf 1971). Although $u_{b}$ is used in Eq. (2) to calculate the instantaneous drag force, using the temporal mean velocity $\bar{u}_{b}$ to evaluate $C_{D}$ is a necessarily simplified treatment of the problem. The lift coefficient is generally considered as an unknown function of $\mathrm{R}_{p}$, although a number of experimental investigations have been carried out (reviewed by Garde and Ranga Raju 1985; Wiberg and Smith 1985). The study of Chepil (1958) provides one of the most complete sets of data, which indicates that the average ratio of lift to drag is nearly constant at 0.85 for boundary Reynolds number $\mathrm{R}^{*} \leqslant 5,000$, where $\mathrm{R}^{*}=u_{*} d^{\prime} / \nu, u_{*}=$ shear velocity $=\sqrt{\tau / \rho}, \tau$ $=$ bed shear stress. This constant ratio of lift to drag was used by Wiberg and Smith (1985) in their theoretical model of particle saltation. The more recent work of Patnaik et al. (1994) appears to demonstrate a decreasing trend of the lift-drag ratio with $\mathrm{R}_{p}$ for their test range of $4,000<R_{p}<60,000$. Their data also show that nearly all the lift-drag ratios (except 1 out of 12 data) are within the range of $0.5-1.5$ for $\mathrm{R}_{p}<8,000$. The aforementioned values of the lift-drag ratio could be useful for the present study because the condition of $\mathrm{R}_{p}<8,000$ (i.e., $\mathrm{R}^{*}<1,000$ ) corresponds to more than $95 \%$ of the situations considered (sample size $=3,000$ ). With the information implied from above, the ratio of lift to drag is taken as unity in this study, which is equivalent to a condition of $C_{L} / C_{D}=1$ given the definitions of drag and lift in Eq. (2). Thus, the lift coefficient becomes an explicit function of $\mathrm{R}_{p}$, as expressed by Eq. (3). However, the negative lift for $R^{*}<5$ reported by Davies and Samad (1978) is not considered herein because the underflow beneath the sphere is assumed negligible for smooth-bed flows (Nikora et al. 2001).

The mean velocity distribution in the wall region (i.e., the inner layer above the viscous sublayer) of the turbulent flow over a hydraulically smooth bed (as the sand bed shown in Fig. 1) can be described by the logarithmic profile (van Rijn 1984; Nezu and Nakagawa 1993)

$$
\frac{\bar{u}}{u_{*}}=\frac{1}{\kappa} \ln \left(\frac{y}{y_{0}}\right)
$$

where $\bar{u}=$ temporal mean velocity at a height $y$ above the bed level; $\kappa=$ von Kármán constant $=0.4$ for clear water; $y_{0}=$ zero-velocity level of the logarithmic profile $=k_{s} / 30$; and $k_{s}=$ equivalent sand roughness of Nikuradse. Note that $y_{0}$ is a virtual zero-velocity level, the real zero-velocity level is located at $y$ $=0$ for smooth-bed flows. The $k_{s}$ value should be set as $0.5 d$ to $3 d$ when Eq. (4) is used in turbulent flow over sand beds (Bridge and Bennett 1992; Ling 1995). Again, to be consistent with the preceding work of this paper (Cheng and Chiew 1998; Wu and Lin 2002), herein we assume $k_{s}=2 d$, which leads to a result of $y_{0}=d / 15$. The area-averaged velocity over the exposed frontal area $A$ is defined by

$$
\bar{u}_{b}=\frac{\int_{A} \bar{u} d A}{\int_{A} d A}
$$

where the differential area $d A=(d \sin \psi) d y$, as illustrated in Fig. 3. Since that $\delta$ is a random variable, for a specific $\delta, \sin \psi$ is given by 


$$
\sin \psi=\sqrt{1-\cos ^{2} \psi}=2 \sqrt{(y-\delta)(d-y+\delta)} / d
$$

where $\cos \psi=[0.5 d-(y-\delta)] / 0.5 d$. Using the definitions of $d A$ and $y_{0}$, and Eqs. (4) and (6), one can rewrite Eq. (5) as the following:

$$
\bar{u}_{b}=\frac{\int_{y_{1}}^{y_{2}}\left[\frac{u_{*}}{\kappa} \ln \left(\frac{15 y}{d}\right)\right] \sqrt{(y-\delta)(d-y+\delta)} d y}{\int_{y_{1}}^{y_{2}} \sqrt{(y-\delta)(d-y+\delta)} d y}
$$

where $y_{1}=0.25 d$ and $y_{2}=d+\delta$ are the lower and upper limits of integration, respectively (shown in Fig. 3). Eq. (7) is derived for a given value of $\delta$, hence $\bar{u}_{b}$ can be expressed as a function of $\delta$. The $\bar{u}_{b}$ value is used to solve Eq. (4) for $y_{b}=y_{0} \times \exp \left(\kappa \bar{u}_{b} / u_{*}\right)$, in which $y_{b}$ is the height (from the bed level) where the mean flow velocity $\bar{u}=\bar{u}_{b}$ (as illustrated in Fig. 1). Because $y_{b}$ is determined from $\bar{u}_{b}$, the $y_{b}$ value so obtained is also a function of $\delta$ and will be used in the derivation of rolling threshold.

\section{Rolling Threshold}

The threshold condition for rolling to begin is that the force moments causing the particle motion exceed those keeping the particle at rest. Such a condition can be expressed as

$$
F_{D} L_{D}+F_{L} L_{L}>W L_{W}
$$

where $L_{D}, L_{L}$, and $L_{W}=$ moment arms (about $C$ ) of $F_{D}, F_{L}$, and $W$ respectively (as shown in Fig. 1). Combination of Eqs. (2) and (8) leads to the following:

$$
u_{b}^{2}>B_{R}^{2}
$$

where $B_{R}$ denotes the rolling threshold and can be expressed by

$$
B_{R}=\sqrt{\frac{2 L_{W}}{C_{D} L_{D}+C_{L} L_{L}} \frac{\pi d^{3}}{6 A} \frac{\gamma_{s}-\gamma}{\rho}}
$$

where $\gamma$ and $\gamma_{s}=$ specific weights of fluid and sediment, respectively. Sphere 1 will start to move in the rolling mode when the inequality in Eq. (9) is satisfied.

As illustrated in Fig. 1, the submerged weight $W$ acts on the center of Sphere 1 in the downward direction. The lift force $F_{L}$ is perpendicular to the flow direction (i.e., in the upward direction); previous studies have generally assumed that $F_{L}$ acts on a line passing through the center of Sphere 1 . The drag force $F_{D}$ acts in the flow direction, however, to date there is no general consensus regarding the position where $F_{D}$ applies on Sphere 1 . It is indicated in the literature that the height of effective drag is normally taken as $0.5 d$ to $0.7 d$ above the bed level (Jackson 1981; Bridge and Bennett 1992), varying with the location selected as the theoretical bed level. The height of effective fluid drag must be a quantity that depends upon the local velocity profile and the protrusion height of the particle. The theoretical value of this height can be obtained through integrations of the local drag and the associated moment over the grain surface (e.g., Jackson 1981), which would not be practical for the present study because the mean drag $F_{D}$ is calculated from the area-averaged velocity $u_{b}$. Nevertheless, the height of mean drag can be evaluated reasonably well with the assumption that $F_{D}$ acts on the level where the mean velocity $\bar{u}=\bar{u}_{b}$, since $\bar{u}_{b}$ is a function of velocity profile and protrusion height. In other words, the $y_{b}$ value gained in the preceding subsection can be taken as the height of effective drag (as shown in Fig. 1), which directly results in $L_{D}=y_{b}-h$. Given that $h=0.125 d+0.5 \delta$, one can evaluate $L_{D}$ by

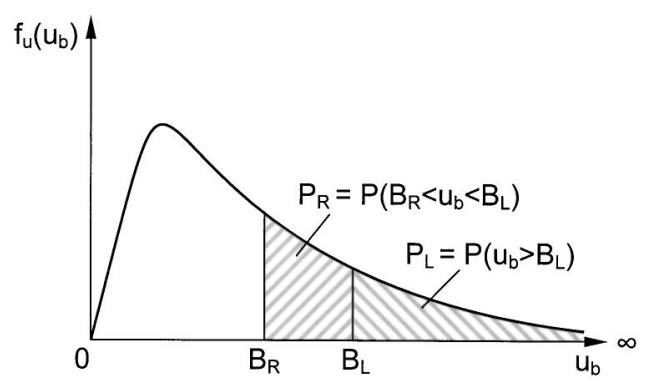

Fig. 4. Schematic diagram showing probability density function of $u_{b}$ and definitions of rolling and lifting probabilities

$$
L_{D}=y_{b}-0.125 d-0.5 \delta
$$

On the other hand, the identical value of $L_{L}$ and $L_{W}$ is equal to $0.5 d \times \sin \phi$. Since that $\cos \phi=(0.5 d+\delta+0.25 d) / d=0.75$ $+(\delta / d)$, one obtains the following:

$$
L_{L}=L_{W}=0.5 d \sqrt{1-[0.75+(\delta / d)]^{2}}
$$

Eqs. (11) and (12) imply that all the moment arms (i.e., $L_{D}, L_{L}$, and $L_{W}$ ) are dependent upon the value of $\delta$.

\section{Lifting Threshold}

The threshold condition for lifting to occur is that the dynamic lift on a sediment particle exceeds its submerged weight, which can be represented by

$$
F_{L}>W
$$

Employing the definitions given in Eq. (2), one can rewrite Eq. (13) as

$$
u_{b}^{2}>B_{L}^{2}
$$

where $B_{L}$ denotes the lifting threshold, which can be expressed as

$$
B_{L}=\sqrt{\frac{2}{C_{L}} \frac{\pi d^{3}}{6 A} \frac{\gamma_{s}-\gamma}{\rho}}
$$

Sphere 1 will initiate its motion in the lifting mode when the criterion given in Eq. (14) is met.

To further compare the rolling and lifting thresholds, we use Eqs. (10) and (15) to evaluate the ratio of the two thresholds. Since $C_{L}=C_{D}$, this ratio becomes

$$
\frac{B_{R}}{B_{L}}=\sqrt{\frac{L_{L}}{L_{D}+L_{L}}}<1
$$

Eq. (16) indicates that the entrainment threshold for lifting is higher than that for rolling, which coincides with the result obtained by Ling (1995). With the two thresholds identified in this section and the probability distribution of the instantaneous velocity to be specified subsequently, the probabilities for entrainment can be defined precisely.

\section{Probabilities for Entrainment}

In a recent comparative study, Wu and Lin (2002) have shown that the instantaneous velocity $u_{b}$ follows the log-normal distribution rather than normal (the pdf of $u_{b}$ is schematically illustrated in Fig. 4). If $v_{b}$ denotes the logarithm of $u_{b}$ (i.e., $v_{b}$ $=\ln u_{b}$ for $\left.0<u_{b}<\infty\right)$, the pdf of $v_{b}$ can be represented by a Gaussian distribution, i.e., 


$$
f_{v}\left(v_{b}\right)=\frac{1}{\sqrt{2 \pi} \sigma_{v}} \exp \left[-\frac{\left(v_{b}-\bar{v}_{b}\right)^{2}}{2 \sigma_{v}^{2}}\right]
$$

where $\bar{v}_{b}$ and $\sigma_{v}=$ mean and standard deviation of $v_{b}$, respectively. Because $u_{b}$ is a non-negative variable, the threshold conditions demonstrated in Eqs. (9) and (14) can be modified as $u_{b}$ $>B_{R}$ for rolling and $u_{b}>B_{L}$ for lifting. Accordingly, if $B_{R}<u_{b}$ $<B_{L}$, the particle will be entrained in a pure rolling mode while still keeping in contact with the bed spheres. However, if $u_{b}$ is greater than $B_{L}$, the incipient motion of the particle will occur in a simultaneous rolling-lifting mode. In other words, the particle is lifted off the bed while it starts to roll. Because the lifted particle is no longer in contact with the spheres below it, herein we identify this type of entrainment (i.e., for $u_{b}>B_{L}$ ) as the lifting mode. Based on these, it is now possible to define the rolling and lifting probabilities.

\section{Rolling Probability}

The probability of entrainment in the rolling mode can be expressed by the following (schematically illustrated in Fig. 4):

$$
\begin{aligned}
P_{R} & =P\left(B_{R}<u_{b}<B_{L}\right)=P\left(B_{R}^{\prime}<v_{b}<B_{L}^{\prime}\right) \\
& =P\left(v_{b}<B_{L}^{\prime}\right)-P\left(v_{b}<B_{R}^{\prime}\right)
\end{aligned}
$$

where $B_{R}^{\prime}$ and $B_{L}^{\prime}=\ln B_{R}$ and $\ln B_{L}$, respectively. Because $v_{b}$ is normally distributed, one can use the approximation presented in Cheng and Chiew (1998) and rewrite Eq. (18) as

$$
\begin{aligned}
P_{R}= & \int_{-\infty}^{B_{L}{ }^{\prime}} f_{v}\left(v_{b}\right) d v_{b}-\int_{-\infty}^{B_{R}{ }^{\prime}} f_{v}\left(v_{b}\right) d v_{b} \\
= & {\left[\int_{-\infty}^{\bar{v}_{b}} f_{v}\left(v_{b}\right) d v_{b}+\int_{\bar{v}_{b}}^{B_{L}{ }^{\prime}} f_{v}\left(v_{b}\right) d v_{b}\right] } \\
& -\left[\int_{-\infty}^{\bar{v}_{b}} f_{v}\left(v_{b}\right) d v_{b}+\int_{\bar{v}_{b}}^{B_{R}{ }^{\prime}} f_{v}\left(v_{b}\right) d v_{b}\right] \\
= & \frac{1}{2}\left\{\frac{B_{L}^{\prime}-\bar{v}_{b}}{\left|B_{L}^{\prime}-\bar{v}_{b}\right|} \sqrt{1-\exp \left[-\frac{2\left(B_{L}^{\prime}-\bar{v}_{b}\right)^{2}}{\pi \sigma_{v}^{2}}\right]}\right. \\
& \left.-\frac{B_{R}^{\prime}-\bar{v}_{b}}{\left|B_{R}^{\prime}-\bar{v}_{b}\right|} \sqrt{1-\exp \left[-\frac{2\left(B_{R}^{\prime}-\bar{v}_{b}\right)^{2}}{\pi \sigma_{v}^{2}}\right]}\right\}
\end{aligned}
$$

in which $\bar{v}_{b}$ and $\sigma_{v}$ can be determined by the following formulas (Wu and Lin 2002):

$$
\begin{gathered}
\bar{v}_{b}=\ln \left[\bar{u}_{b} / \sqrt{1+\left(\sigma_{u} / \bar{u}_{b}\right)^{2}}\right] \\
\sigma_{v}^{2}=\ln \left[1+\left(\sigma_{u} / \bar{u}_{b}\right)^{2}\right]
\end{gathered}
$$

where $\sigma_{u}=$ standard deviation of $u_{b}$. According to Kironoto and Graf (1994) and Cheng and Chiew (1998), a linear relationship between $\sigma_{u}$ and $\bar{u}_{b}$ is given by $\sigma_{u}=0.37 \bar{u}_{b}$, which transforms Eq. (20) into

$$
\begin{gathered}
\bar{v}_{b}=\ln \left(\bar{u}_{b} / 1.066\right) \\
\sigma_{v}^{2}=0.128
\end{gathered}
$$

To proceed with the derivation of rolling probability, we rewrite Eqs. (7), (10), and (15) as the following:

$$
\begin{gathered}
\bar{u}_{b}=\frac{u_{*} \times \int_{y_{1}}^{y_{2}}\left[\frac{1}{\kappa} \ln \left(\frac{15 y}{d}\right)\right] \sqrt{(y-\delta)(d-y+\delta)} d y}{\int_{y_{1}}^{y_{2}} \sqrt{(y-\delta)(d-y+\delta)} d y}=u_{*} \times H \\
B_{R}=\sqrt{\frac{2 L_{W}}{C_{D} L_{D}+C_{L} L_{L}} \frac{\pi d^{2}}{6 A} \frac{\left(\gamma_{s}-\gamma\right) d}{\rho}}=\sqrt{J_{R} \times K \frac{\left(\gamma_{s}-\gamma\right) d}{\rho}} \\
B_{L}=\sqrt{\frac{2}{C_{L}} \frac{\pi d^{2}}{6 A} \frac{\left(\gamma_{s}-\gamma\right) d}{\rho}}=\sqrt{J_{L} \times K \frac{\left(\gamma_{s}-\gamma\right) d}{\rho}}
\end{gathered}
$$

where $H=$ dimensionless function of $\delta$ as given in Eq. (22); while $K=\pi d^{2} / 6 A, J_{R}=2 L_{W} /\left(C_{D} L_{D}+C_{L} L_{L}\right)$, and $J_{L}=2 / C_{L}$ are all dimensionless and varying with $\delta$. Substituting Eqs. (21)-(24) into Eq. (19) results in

$$
\begin{aligned}
P_{R}(\delta)= & 0.5 \frac{\ln \left(1.137 J_{L} K / H^{2} \theta\right)}{\left|\ln \left(1.137 J_{L} K / H^{2} \theta\right)\right|} \\
& \times \sqrt{1-\exp \left\{-\frac{2}{\pi}\left[\frac{\ln \left(1.137 J_{L} K / H^{2} \theta\right)}{0.358}\right]^{2}\right\}} \\
& -0.5 \frac{\ln \left(1.137 J_{R} K / H^{2} \theta\right)}{\left|\ln \left(1.137 J_{R} K / H^{2} \theta\right)\right|} \\
& \times \sqrt{1-\exp \left\{-\frac{2}{\pi}\left[\frac{\ln \left(1.137 J_{R} K / H^{2} \theta\right)}{0.358}\right]^{2}\right\}}
\end{aligned}
$$

where $P_{R}(\delta)=$ rolling probability for a given $\delta ; \theta=$ dimensionless shear stress (or Shields parameter) defined as $\tau /\left(\gamma_{s}-\gamma\right) d$. Recall that $\delta$ is a uniform random variable between $-0.75 d$ and $0.116 d$, the mean probability of rolling $(P R)$ is simply the expected value of Eq. (25), i.e.,

$$
\begin{aligned}
P R & =\int_{-0.75 d}^{0.116 d} P_{R}(\delta) f_{\Delta}(\delta) d \delta=\frac{1}{0.866 d} \int_{-0.75 d}^{0.116 d} P_{R}(\delta) d \delta \\
& =\frac{1}{0.866} \int_{-0.75}^{0.116} P_{R}\left(\delta^{\prime}\right) d \delta^{\prime}
\end{aligned}
$$

where $\delta^{\prime}=\delta / d=$ dimensionless dummy variable, and $P_{R}\left(\delta^{\prime}\right)=$ nondimensionalized form of Eq. (25). Eq. (26) is then solved numerically for evaluating the rolling probabilities corresponding to a range of $\theta$ (between $10^{-2}$ and $10^{1}$ in this study).

\section{Lifting Probability}

The probability of entrainment in the lifting mode can be expressed as the following (illustrated in Fig. 4):

$$
P_{L}=P\left(u_{b}>B_{L}\right)=P\left(v_{b}>B_{L}^{\prime}\right)=1-P\left(-\infty<v_{b}<B_{L}^{\prime}\right)
$$

Following the same procedures used in Eq. (19), one can reform Eq. (27) as

$$
\begin{aligned}
P_{L} & =1-\left[\int_{-\infty}^{\bar{v}_{b}} f_{v}\left(v_{b}\right) d v_{b}+\int_{\bar{v}_{b}}^{B_{L}{ }^{\prime}} f_{v}\left(v_{b}\right) d v_{b}\right] \\
& =0.5-0.5 \frac{B_{L}^{\prime}-\bar{v}_{b}}{\left|B_{L}^{\prime}-\bar{v}_{b}\right|} \sqrt{1-\exp \left[-\frac{2\left(B_{L}^{\prime}-\bar{v}_{b}\right)^{2}}{\pi \sigma_{v}^{2}}\right]}
\end{aligned}
$$

Similarly, substituting Eqs. (21), (22), and (24) into Eq. (28) yields 


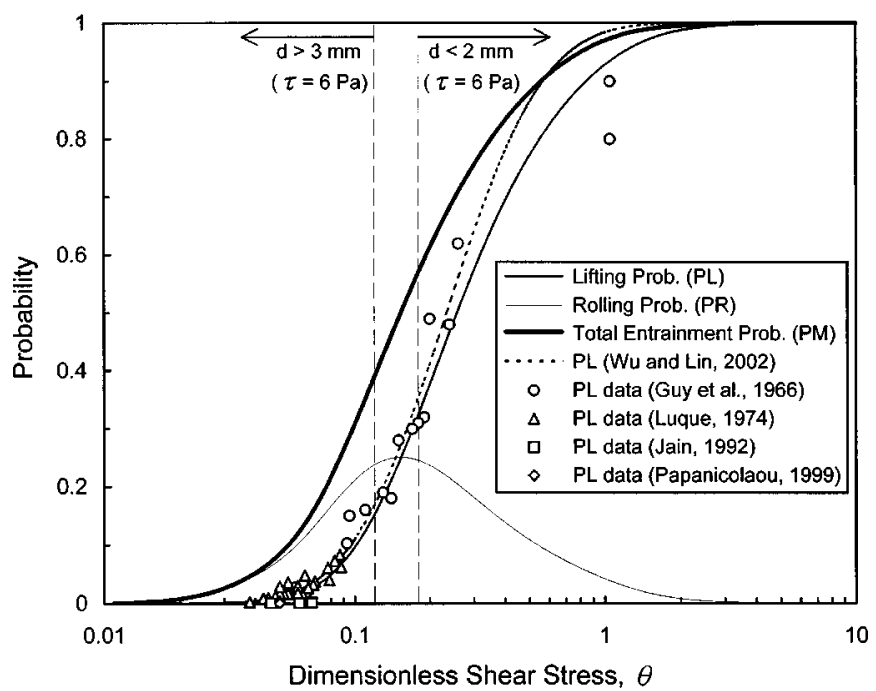

Fig. 5. Relationships between entrainment probabilities and dimensionless shear stress

$$
\begin{aligned}
P_{L}(\delta)= & 0.5-0.5 \frac{\ln \left(1.137 J_{L} K / H^{2} \theta\right)}{\left|\ln \left(1.137 J_{L} K / H^{2} \theta\right)\right|} \\
& \times \sqrt{1-\exp \left\{-\frac{2}{\pi}\left[\frac{\ln \left(1.137 J_{L} K / H^{2} \theta\right)}{0.358}\right]^{2}\right\}}
\end{aligned}
$$

where $P_{L}(\delta)=$ lifting probability for a specific value of $\delta$. The mean probability of lifting $(P L)$ is taken as the expected value of Eq. (29), i.e.,

$$
P L=\int_{-0.75 d}^{0.116 d} P_{L}(\delta) \cdot f_{\Delta}(\delta) d \delta=\frac{1}{0.866} \int_{-0.75}^{0.116} P_{L}\left(\delta^{\prime}\right) d \delta^{\prime}
$$

where $P_{L}\left(\delta^{\prime}\right)=$ nondimensionalized form of Eq. (29). Eq. (30) is also solved numerically to evaluate the lifting probabilities corresponding to a range of $\theta$ between $10^{-2}$ and $10^{1}$.

\section{Results and Discussion}

\section{Verification of Results}

The relationships between the computed results and the dimensionless shear stress $\theta$ are demonstrated in Fig. 5, where one can see the distinct difference between the lifting and rolling probabilities. The lifting probability $P L$ increases monotonously with $\theta$, whereas the rolling probability $P R$ increases with $\theta$ in the region of $\theta<0.15$ but then reduces for larger values of $\theta$. In what follows, we verify these results with the published data. First, the lifting probabilities reported by Guy et al. (1966), Luque (1974), Jain (1992), and Papanicolaou (1999) are used for comparison with the calculated $P L$. Fig. 5 reveals that the computed result of $P L$ agrees generally well with the published data. The discrepancies present at the upper end are probably due to the observed flow-retardation (or drag-reduction) effect caused by the impact on the near-bed flow of the increasing particles in motion at higher $\theta$ values (McEwan 2002). The lifting probability from $\mathrm{Wu}$ and Lin (2002) is also presented in Fig. 5 to demonstrate the improvement made in the current study. The earlier result of $\mathrm{Wu}$ and Lin (2002) displays a substantial overestimation of lifting
Table 1. Euclidean Norms and Coefficients of Determination for Results from Different Studies (Percentage of Change from Earlier Result is in Brackets)

\begin{tabular}{lcc}
\hline Source & $\begin{array}{c}\text { Previous study } \\
(\text { Wu and Lin 2002) }\end{array}$ & This study \\
\hline$\|\mathrm{e}\|_{2}$ & 0.266 & $0.245[-8 \%]$ \\
$R^{2}$ & 0.966 & $0.971[+0.5 \%]$ \\
\hline
\end{tabular}

probability for $\theta \approx 1$. To quantitatively explore the improved accuracy, the Euclidean norm is used as a measure to represent the overall error between the theoretical and experimental results (Conte and de Boor 1980). The Euclidean norm is defined as

$$
\|\mathrm{e}\|_{2}=\sqrt{\sum_{i=1}^{n} \mathrm{e}_{i}^{2}}
$$

where $\mathrm{e}_{i}=$ difference between the $i$ th data and the theoretical value; $n=$ total number of the experimental data. The magnitudes of the Euclidean norm and the coefficient of determination $R^{2}$ for the earlier result of $\mathrm{Wu}$ and $\mathrm{Lin}$ (2002) and the present result are listed in Table 1 . The values of $\|\mathrm{e}\|_{2}$ and $R^{2}$ for the result of $\mathrm{Wu}$ and Lin are 0.266 and 0.966 , respectively, whereas the corresponding values for the present result are 0.245 and 0.971 . The percentages of the reduced $\|\mathrm{e}\|_{2}$ and the improved $R^{2}$ are about 8 and $0.5 \%$, respectively. In contrast to the previous work of $\mathrm{Wu}$ and Lin (2002), the present study incorporates the probabilistic feature of the initial bed geometry (in addition to the turbulent velocity fluctuation) and also the dependence of lift coefficient on flow condition (whereas a constant lift coefficient $C_{L}=0.21$ was used by $\mathrm{Wu}$ and Lin). The improved accuracy is believed to originate from these additional considerations.

Second, the rolling and lifting probabilities are compared with the qualitative observations of Drake et al. (1988). Their observations were made by motion-picture photography at Duck Creek, a clear stream $6.5 \mathrm{~m}$ wide and $35 \mathrm{~cm}$ deep near Pinedale, Wyoming. Bed-shear stress was approximately $6 \mathrm{~Pa}$. The streambed is hydraulically transitional, consisting of fine gravels with a median diameter of $4 \mathrm{~mm}$. The transport of sediment was almost entirely as bed load. The recorded plan and side views of the motion of individual bed load particles indicated that rolling was the most common mode of entrainment for particles larger than about 3 $\mathrm{mm}$, whereas lifting was the mode of entrainment for most bed load particles smaller than about $2 \mathrm{~mm}$. The reported bed-shear stress (i.e., $6 \mathrm{~Pa}$ ) and particle diameters correspond to the values of $\theta=0.12$ (for $d=3 \mathrm{~mm}$ ) and $\theta=0.18$ (for $d=2 \mathrm{~mm}$ ), respectively. In other words, when $\theta<0.12$, rolling is the commonest mode of entrainment, whereas for $\theta>0.18$, lifting is the dominant mode of entrainment. Clearly demonstrated in Fig. 5 is that the rolling probability is greater than the lifting probability in the region of $\theta<0.12$; however, the lifting probability becomes much greater than the rolling probability for $\theta>0.18$. The results obtained in this study coincide very well with the observations made by Drake et al. (1988) and are physically meaningful. In fact, such a coincidence can be reasonably interpreted because when the values of $\theta$ are sufficiently high (i.e., for very large $\tau$ values or very small $d$ values), there is a strong tendency that particles will be entrained in the lifting (i.e., rolling-lifting) mode rather than the pure rolling mode. On the other hand, when the magnitudes of $\theta$ are appreciably low (i.e., for negligible $\tau$ values or extremely large $d$ values), the particles will most likely stay in repose rather than move. As such, the probability of entrainment in the rolling mode becomes vanishing small at both very high and low values of $\theta$. 


\section{Total Probability of Incipient Motion}

As mentioned earlier, various forms of entrainment probabilities (such as rolling, lifting, and sliding probabilities) have been used in the stochastic bed load models. We have come to know that the entrainment of sediment occurs mainly in the rolling and lifting modes. It is revealed in Fig. 5 that for a given value of $\theta$, there are always two possible modes of entrainment, regardless of which one is more likely to occur. Thus, instead of using a single rolling or lifting probability, it is more reasonable to use the total probability of entrainment (inclusive of both rolling and lifting) in the modeling of bed load transport. Because rolling and lifting are mutually independent modes according to our definition (as illustrated in Fig. 4), the total probability of incipient motion $\left(P_{M}\right)$ is equal to the summation of rolling and lifting probabilities, i.e., $P_{M}=P_{R}+P_{L}$. Taking the expected value of $P_{M}$ over the entire range of $\delta$ yields

$$
P M=P R+P L
$$

where $P M=$ mean total probability of entrainment. The $P M$ curve resulting from the superimposition of $P R$ and $P L$ curves is shown in Fig. 5. It is found that the rolling probability makes up more than $90 \%$ of the total entrainment probability for any $\theta$ value less than about 0.05 , while the lifting probability occupies more than $90 \%$ of the total entrainment probability for any $\theta$ value greater than about 0.6 . Hence, for the regions of $\theta<0.05$ and $\theta>0.6, P R$ and $P L$ can be used, respectively, as the approximations to $P M$. However, for the $\theta$ values in the range between 0.05 and 0.6 , the contributions of both probabilities to the total probability of entrainment should be equally weighted.

\section{Potential Applications}

The entrainment probabilities developed in this study are applicable to many aspects involving the predictions of bed load movement in natural channels. For example, the stochastic bed load models based on the single-mode entrainment probabilities, such as those of Einstein (1950), Paintal (1971), and Sun and Donahue (2000), can be modified using the total entrainment probability. Given the multiple modes associated with the initial motion of bed load particles, it is not surprising that some of the predictions based on a single entrainment mode have displayed considerable errors. The discrepancies between the predicted and measured results are often reduced through the calibration procedures. However, the modifications resulting from such procedures can be rather limited. Hence, a significant improvement may be expected if a more reliable entrainment probability is incorporated into the stochastic modeling of bed load transport.

In addition, the partitioning approach for the sediment entrainment into a rolling and lifting mode can be useful to the river managers in planning of the flushing flows for restoring the salmonid incubation habitat degraded by fine sediment intrusion (Wu 2000). For the flushing flows to be effective, the removal of fine matrix material should be enhanced to improve the quality of aquatic habitat as much as possible, whereas the movement of coarse framework material should be limited to reduce the loss of coarse material and to maintain a stable framework of habitat for the incubating embryos. The proposed entrainment probability versus Shields parameter relations may be used to determine the optimal flushing flow so that fine sediment can be lifted off the bed without causing significant disturbances to the coarse framework material. Further studies are currently undertaken by the writers to address this challenging issue.

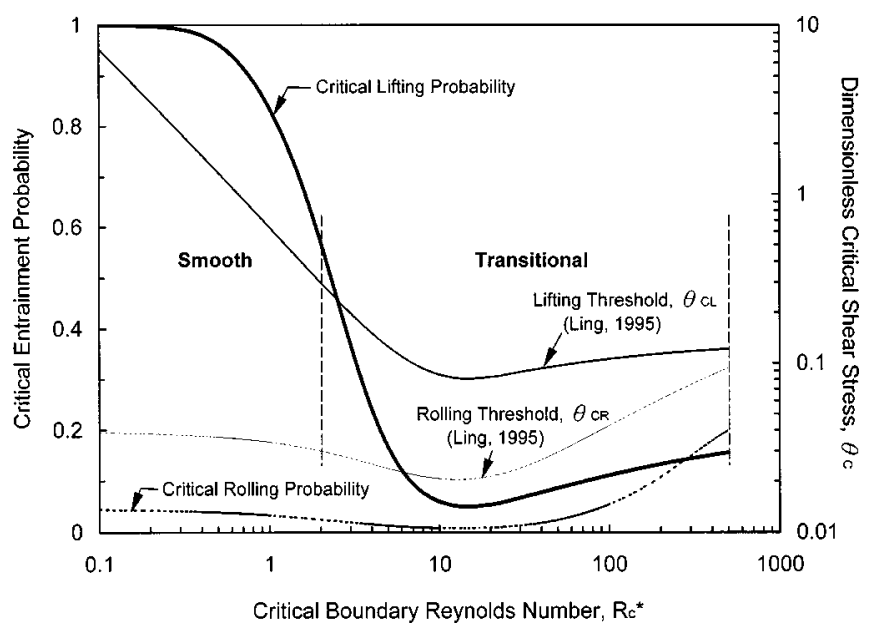

Fig. 6. Variations of dimensionless critical shear stresses and critical entrainment probabilities with critical boundary Reynolds number

\section{Critical Entrainment Probabilities}

So far we have acquired the relations quantifying the variations of $P R$ and $P L$ with $\theta$. Further, we are interested in the probability of entrainment corresponding to the condition that the applied shear stress equals to the threshold shear stress for incipient motion, i.e., $\theta=\theta_{c}$, in which $\theta_{c}=$ dimensionless critical shear stress. Gessler (1971) reported a 50\% probability of movement in rough turbulent flow when $\theta_{c}$ (based on $d_{50}$ ) was applied to the bed particles. The entrainment probabilities at the critical conditions can be evaluated with the aid of the rolling and lifting thresholds developed by Ling (1995). His criteria for incipient motion can be presented in a graphical format similar to Shields diagram, i.e., $\theta_{c}$ versus critical boundary Reynolds number $R_{c}^{*}$ (as shown in Fig. 6). He found that the Shields curve for the most part lies between the two theoretical thresholds. For a given value of $\mathrm{R}_{c}^{*}$, the corresponding rolling and lifting thresholds (i.e., $\theta_{c R}$ and $\theta_{c L}$ ) can be determined from the two separate criteria for incipient motion. The values of $\theta_{c R}$ and $\theta_{c L}$ are then incorporated with the proposed $P R-\theta$ and $P L-\theta$ relations (or Fig. 5) to evaluate the critical rolling and lifting probabilities, respectively. The results so obtained are shown in Fig. 6, where the entrainment probabilities corresponding to the critical conditions demonstrate considerable variations in the magnitude, especially for the critical lifting probability. The maximum and minimum values of the critical lifting probability are 1.0 and 0.05 , respectively, while the critical rolling probability ranges from 0.008 to 0.2 . The critical lifting probability drops drastically from about 0.8 to 0.05 as $R_{c}^{*}$ increases from 1 to 10 . For $R_{c}^{*}>10$, the critical lifting probability increases modestly from 0.05 to 0.16 . On the other hand, the critical rolling probability remains approximately constant within the range between 0.01 and 0.04 for $R_{c}^{*}<10$, but then increases to about 0.2 as $R_{c}^{*}$ increases from 10 to 500 . For hydrodynamically smooth boundaries $\left(\mathrm{R}_{c}^{*}<2\right)$, both of the critical entrainment probabilities display decreasing trends with the increasing $\mathrm{R}_{c}^{*}$. However, in the transitional regime $\left(2<\mathrm{R}_{c}^{*}\right.$ $<500$ ), both of the critical entrainment probabilities demonstrate transitions from descending to ascending trends. These trends of variation appear to correlate with the criteria for incipient motion. Note that in Fig. 6 the rolling threshold and the critical rolling probability are nearly parallel over the entire range of $\mathrm{R}_{c}^{*}$ despite that they are in different graphical scales (i.e., logarithmic and linear scales, respectively). In summary, the probabilities of en- 
trainment corresponding to the critical rolling and lifting conditions are neither constant values nor monotonous functions of $\mathrm{R}_{c}^{*}$. Their variation trends agree with those of the entrainment thresholds.

A comment on the results is given below. If the critical shear stress is a distinct threshold for incipient motion of the sediment, there must be a consistent probability of entrainment corresponding to such a critical condition. For example, a $50 \%$ probability of movement at the critical conditions as proposed by Gessler (1971). However, the results gained in the present study do not support such an argument, in terms of both rolling and lifting modes of entrainment. It is revealed that even when the threshold shear stress is applied to the sediment particle, the entrainment probability is a highly variable function of the hydrodynamic boundary condition, rather than a meaningful value representing the critical state of particle entrainment. Since the probabilities of entrainment corresponding to the so called "critical conditions" vary over such a wide range, a possible explanation would be that there is no such thing as "critical shear stress," as pointed out by many investigators (see review by McEwan and Heald 2001). The results of this study appear to imply the inconsistency embedded in the conventional definition of the critical shear stress for incipient motion, thus probably provide a different perspective worth further investigations.

\section{Conclusions}

In this paper, we present the theoretical formulation of the rolling and lifting probabilities for sediment entrainment in hydraulically smooth and transitional flows. The stochastic natures of turbulent fluctuation and bed grain irregularity are both considered in the present study. The results obtained herein represent an extended version of the earlier pickup probabilities (Cheng and Chiew 1998; Wu and Lin 2002) that only considered the fluctuation of turbulent flow. Modification of the previous studies is also made with the inclusion of a relation between lift coefficient and particle Reynolds number. However, the effect of turbulent bursting has not been included in this paper. It is believed that the entrainment of sediment is sensitive to the periodic burst evens. To include the bursting effect explicitly in future formulation of the entrainment probability would be a challenging task. In this study, the theoretically identified thresholds for entrainment make it possible to precisely define the rolling and lifting probabilities. The results show that the lifting probability (ranging from 0 to 1 ) increases monotonously with the dimensionless shear stress $\theta$, whereas the rolling probability (ranging from 0 to 0.25 ) displays an increasing trend for $\theta<0.15$ yet a decreasing trend for larger $\theta$ values. Both of the rolling and lifting probabilities coincide well with the published data, quantitatively and qualitatively. Moreover, the lifting probability gained in this study demonstrates an improved agreement with the experimental data. For $0.05<\theta$ $<0.6$, the summation of rolling and lifting probabilities is recommended for use as the total probability of incipient motion. However, for $\theta$ less than 0.05 (or greater than 0.6), the rolling (or lifting) probability can be used as the approximation to the total entrainment probability. The critical entrainment probabilities are highly variable functions of the boundary Reynolds number, thus no consistent probability corresponding to the critical state of particle entrainment can be found. The results of this study appear to imply the inconsistency involved in the conventional definition of critical shear stress. Future research is needed to further address this issue and to investigate the influences of turbulence (e.g., intensity, bursting), sediment characteristics (e.g., shape, size distribution, availability or abundance, number of particles in motion), bed geometry (e.g., slope, roughness), and other random factors on the probabilities of sediment entrainment.

\section{Acknowledgments}

Funding of this research was granted by the National Science Council, Republic of China (NSC-90-2211-E-002-085). The writers appreciate the insightful comments and constructive suggestions offered by three anonymous reviewers, which have been incorporated into the final version of this paper.

\section{Notation}

The following symbols are used in this paper:

$A=$ frontal area exposed to the flow;

$B_{L}, B_{R}=$ lifting and rolling thresholds defined by Eqs. (15) and (10);

$B_{L}^{\prime}, B_{R}^{\prime}=\ln B_{L}$ and $\ln B_{R}$;

$C_{D}, C_{L}=$ drag and lift coefficients;

$d=$ diameter of particle;

$d^{\prime}=$ exposed diameter of particle;

$d_{50}=$ median grain size;

$\|\mathrm{e}\|_{2}=$ Euclidean norm of error;

$F_{D}, F_{L}=$ instantaneous drag and lift forces;

$f_{v}\left(v_{b}\right)=$ probability density function (pdf) of $v_{b}$;

$f_{\Delta}(\delta)=$ probability density function (pdf) of $\delta$;

$g=$ gravitational acceleration;

$H=$ dimensionless function of $\delta$;

$h=$ vertical distance from bed level to point of contact $C$;

$J_{L}=2 / C_{L}$

$J_{R}=2 L_{W} /\left(C_{D} L_{D}+C_{L} L_{L}\right)$;

$K=\pi d^{2} / 6 A$

$k_{s}=$ equivalent roughness height of Nikuradse;

$L_{D}, L_{L}, L_{W}=$ moment arms (about $C$ ) of $F_{D}, F_{L}$, and $W$;

$P(X)=$ probability of event $X$;

$P_{L}(\delta), P_{R}(\delta)$

$=$ lifting and rolling probabilities for given $\delta$;

$P_{L}\left(\delta^{\prime}\right), P_{R}\left(\delta^{\prime}\right)$

$=$ nondimensionalized forms of $P_{L}(\delta)$ and $P_{R}(\delta)$;

$P_{M}$ or $P_{M}(\delta)$

$=$ total probability of incipient motion (for a given $\delta$ );

$P L, P R=$ mean probabilities of lifting and rolling;

$P M=$ mean total probability of entrainment;

$\mathrm{R}_{p}=$ particle Reynolds number $=\bar{u}_{b} d^{\prime} / \nu$;

$\mathrm{R}^{*}=$ boundary Reynolds number $=u_{*} d^{\prime} / v$;

$\mathrm{R}_{c}^{*}=$ critical boundary Reynolds number;

$R^{2}=$ coefficient of determination;

$u_{b}=$ area-averaged instantaneous velocity approaching particle;

$u_{*}=$ shear velocity $=\sqrt{\tau / \rho}$;

$\bar{u}=$ temporal mean velocity at height $y$ above bed level;

$\bar{u}_{b}=$ area-averaged mean flow velocity;

$v_{b}=\ln u_{b}$

$\bar{v}_{b}=$ mean value of $v_{b}$;

$W=$ submerged weight of sediment particle; 


$$
\begin{aligned}
x= & \text { coordinates in longitudinal direction; } \\
y= & \text { coordinates in vertical direction; } \\
y_{b}= & \text { height from bed level where } \bar{u}=\bar{u}_{b} ; \\
y_{0}= & \text { zero-velocity level; } \\
y_{1}, y_{2}= & \text { lower and upper limits of integration in Eq. } \\
& (7) ; \\
z= & \text { coordinates in transverse direction; } \\
\gamma, \gamma_{s}= & \text { specific weights of fluid and sediment; } \\
\delta= & \text { vertical distance from bed level to bottom } \\
& \text { of Sphere } 1 ; \\
\delta^{\prime}= & \delta / d ; \\
\theta= & \text { dimensionless shear stress (Shields param- } \\
& \text { eter })=\tau /\left(\gamma_{s}-\gamma\right) d ; \\
\theta_{c}= & \text { dimensionless critical shear stress; } \\
\theta_{c L}, \theta_{c R}= & \text { dimensionless threshold shear stresses for } \\
& \text { lifting and rolling; } \\
\kappa= & \text { von Kármán constant; } \\
v= & \text { kinematic viscosity of fluid; } \\
\rho, \rho_{s}= & \text { densities of fluid and sediment; } \\
\sigma_{u}, \sigma_{v}= & \text { standard deviations of } u_{b} \text { and } v_{b} ; \text { and } \\
\tau= & \text { bed shear stress. }
\end{aligned}
$$

\section{References}

Bridge, J. S., and Bennett, S. J. (1992). "A model for the entrainment and transport of sediment grains of mixed sizes, shapes, and densities." Water Resour. Res., 28(2), 337-363.

Buffington, J. M., Dietrich, W. E., and Kirchner, J. W. (1992). "Friction angle measurements on a naturally formed gravel streambed - implications for critical boundary shear-stress." Water Resour. Res., 28(2), 411-425.

Buffington, J. M., and Montgomery, D. R. (1997). "A systematic analysis of eight decades of incipient motion studies, with special reference to gravel-bedded rivers." Water Resour. Res., 33(8), 1993-2029.

Cheng, N.-S., and Chiew, Y.-M. (1998). "Pickup probability for sediment entrainment." J. Hydraul. Eng., 124(2), 232-235.

Cheng, N.-S., and Chiew, Y.-M. (1999). "Closure to discussion of 'Pickup probability for sediment entrainment'." J. Hydraul. Eng., 125(7), 789-789.

Chepil, W. S. (1958). "The use of evenly spaced hemispheres to evaluate aerodynamic forces on a soil surface." Trans., Am. Geophys. Union, 39(3), 397-404.

Church, M. A. (1978). "Palaeohydrological reconstructions from a Holocene valley fill." Fluvial sedimentology, A. D. Miall, ed., Canadian Society of Petroleum Geologists, Alberta, Canada, 743-772.

Conte, S. D., and de Boor, C. (1980). Elementary numerical analysis, McGraw-Hill, New York.

Davies, T. R. H., and Samad, M. F. A. (1978). "Fluid dynamic lift on a bed particle." J. Hydraul. Div., Am. Soc. Civ. Eng., 104, 1171-1182.

Drake, T. G., Shreve, R. L., Dietrich, W. E., Whiting, P. J., and Leopold, L. B. (1988). "Bedload transport of fine gravel observed by motionpicture photography." J. Fluid Mech., 192, 193-217.

Einstein, H. A. (1942). "Formula for the transportation of bed load." Trans. Am. Soc. Civ. Eng., 107, 561-597.

Einstein, H. A. (1950). "The bed load function for sediment transportation in open channel flows." Tech. Bull. 1026, USDA, Washington, D.C.

Garde, R. J., and Ranga Raju, K. G. (1985). Mechanics of sediment transportation and alluvial stream problems, Wiley Eastern, New Delhi, India.

Gessler, J. (1971). "Beginning and ceasing of sediment motion." River mechanics, H. W. Shen, ed., Fort Collins, Colo., 7:1-7:22.

Graf, W. H. (1971). Hydraulics of sediment transport. McGraw-Hill, New York.
Grass, A. J. (1970). "Initial instability of fine sand." J. Hydraul. Div., Am. Soc. Civ. Eng., 96(3), 619-632.

Guy, H. P., Simons, D. B., and Richardson, E. V. (1966). "Summary of alluvial channel data from flume experiments, 1956-1961." USGS Professional Paper.

Halow, J. S. (1973). "Incipient rolling, sliding and suspension of particles in horizontal and inclined turbulent flow." Chem. Eng. Sci., 28, 1-12.

Jackson, P. S. (1981). "On the displacement height in the logarithmic velocity profile." J. Fluid Mech., 111, 15-25.

Jain, S. C. (1992). "Note on lag in bedload discharge." J. Hydraul. Eng., 118(6), 904-917.

Kalinske, A. A. (1947). "Movement of sediment as bed load in rivers." Trans., Am. Geophys. Union, 4, 310-317.

Kirchner, J. W., Dietrich, W. E., Iseya, F., and Ikeda, H. (1990). "The variability of critical shear-stress, friction angle and grain protrusion in water-worked sediments." Sedimentology, 37(4), 647-672.

Kironoto, B. A., and Graf, W. H. (1994). "Turbulence characteristics in rough uniform open-channel flow." Proc., Inst. Civ. Eng., Waters, Maratime Energ., 106(Dec), 333-344.

Lavelle, J. W., and Mofjeld, H. O. (1987). "Do critical stresses for incipient motion and erosion really exist?" J. Hydraul. Eng., 113(3), 370-385.

Ling, C.-H. (1995). "Criteria for incipient motion of spherical sediment particles.” J. Hydraul. Eng., 121(6), 472-478.

Luque, R. F. (1974). Erosion and transport of bed load sediment, Delft Univ. of Technology, Delft, The Netherlands.

McEwan, I. (2002). "Discussion of "Flow turbulence over fixed and weakly mobile gravel beds." J. Hydraul. Eng., 128(3) (359-360).

McEwan, I., and Heald, J. (2001). "Discrete particle modeling of entrainment from flat uniformly sized sediment beds." J. Hydraul. Eng., 127(7), 588-597.

Meyer-Peter, E., and Müller, R. (1948). "Formulas for bed-load transport." Proc., 2nd IAHR Congress, Stockholm, Sweden, 39-64.

Naden, P. (1987). "An erosion criterion for gravel-bed rivers." Earth Surf. Processes Landforms, 12, 83-93.

Nezu, I., and Nakagawa, H. (1993). Turbulence in open-channel flows, A. A. Balkema, Rotterdam, The Netherlands.

Nikora, V. I., Goring, D. G., and Biggs, B. J. F. (1998). "On gravel-bed roughness characterization." Water Resour. Res., 34(3), 517-527.

Nikora, V. I., Goring, D. G., McEwan, I., and Griffiths, G. (2001). "Spatially averaged open-channel flow over rough bed." J. Hydraul. Eng., 127(2), 123-133.

Paintal, A. S. (1971). "A stochastic model of bed-load transport." $J$. Hydraul. Res., 9(4), 527-554.

Papanicolaou, A. N. (1999). "Discussion of "pickup probability for sediment entrainment'." J. Hydraul. Eng., 125(7), 788-789.

Papanicolaou, A. N., Diplas, P., Dancey, C. L., and Balakrishnan, M. (2001). "Surfce roughness effects in near-bed turbulence: Implications to sediment entrainment." J. Eng. Mech., 127(3), 211-218.

Papanicolaou, A. N., Diplas, P., Evaggelopoulos, N., and Fotopoulos, S., (2002). "Stochastic incipient motion criterion for spheres under various bed packing conditions." J. Hydraul. Eng., 128(4), 369-380.

Parker, G. (1979). "Hydraulic geometry of active gravel rivers." J. Hydraul. Div., Am. Soc. Civ. Eng., 105(9), 1185-1201.

Patnaik, P. C., Vittal, N., and Pande, P. K. (1994). "Lift coefficient of a stationary sphere in gradient flow." J. Hydraul. Res., 32(3), 471-480.

Schiller, L., and Naumann, A. (1933). "Uber die grundlegenden berechnungen bei der schwerdraftaufbereitung." Zeitschrift Des verein deutscher Ingenieure, Düsseldorf, Germany, 77, 318-320 (in German).

Shields, A. (1936). "Application of similarity principles and turbulence research to bed-load movement." Rep., No. 167, California Institute of Technology, Pasadena, Calif. (translated from German).

Sun, Z., and Donahue, J. (2000). "Statistically derived bedload formula for any fraction of nonuniform sediment." J. Hydraul. Eng., 126(2), $105-111$.

van Rijn, L. C. (1984). "Sediment transport. I: Bed load transport." J. Hydraul. Eng., 110(10), 1431-1456. 
Wiberg, P. L., and Smith, J. D. (1985). "A theoretical model for saltating grains in water." J. Geophys. Res. B, 90(C4), 7341-7354.

Wilcock, P. R., Barta, A. F., Shea, C. C., Kondolf, G. M., Matthews, W. V. G., and Pitlick, J. (1996). "Observations of flow and sediment entrainment on a large gravel-bed river." Water Resour. Res., 32(9), $2897-$ 2909.
Wu, F.-C. (2000). "Modeling embryo survival affected by sediment deposition into salmonid spawning gravels: Application to flushing flow prescriptions." Water Resour. Res., 36(6), 1595-1603.

Wu, F.-C., and Lin, Y.-C. (2002). "Pickup probability of sediment under log-normal velocity distribution." J. Hydraul. Eng., 128(4), 438-442.

Wu, W., Wang, S. S. Y., and Jia, Y. (2000). "Nonuniform sediment transport in alluvial rivers." J. Hydraul. Res., 38(6), 427-434. 